\title{
Detection of emerging ibrutinib resistance by flow cytometry in patients with chronic lymphocytic leukemia
}

\section{Ferenc Takács}

Semmelweis University: Semmelweis Egyetem

\section{Lili Kotmayer}

Semmelweis University: Semmelweis Egyetem

Ágnes Czeti

Semmelweis University: Semmelweis Egyetem

\section{Gábor Szalóki}

Semmelweis University: Semmelweis Egyetem

\section{László Tamás}

Semmelweis University: Semmelweis Egyetem

\section{Gábor Mikala}

South-Pest Central Hospital

\section{Ágnes Márk}

Semmelweis University: Semmelweis Egyetem

\section{András Masszi}

National Institute of Oncology: Orszagos Onkologiai Intezet

\section{Péter Farkas}

Semmelweis University: Semmelweis Egyetem

\section{Márk Plander}

Markusovszky University Teaching Hospital

\section{Júlia Weisinger}

Semmelweis University: Semmelweis Egyetem

\section{Judit Demeter}

Semmelweis University: Semmelweis Egyetem

\section{Sándor Fekete}

South-Pest Centrum hospital

\section{Erika Szaleczki}

National Institute of Oncology: Orszagos Onkologiai Intezet

\section{Zita Borbényi}

University Szeged

\section{László Szerafin}


Hospitals of Szabolcs-Szatmár-Bereg County and University Teaching Hospital

\section{Beáta Deák}

National Institute of Oncology: Orszagos Onkologiai Intezet

\section{Adrienn Sulák}

University of Szeged: Szegedi Tudomanyegyetem

Gabor Barna ( $\nabla$ barna.gabor@med.semmelweis-univ.hu )

Semmelweis University: Semmelweis Egyetem https://orcid.org/0000-0002-8895-3062

\section{Research}

Keywords: chronic lymphocytic leukemia, flow cytometry, targeted therapy, ibrutinib, drug resistance

Posted Date: November 15th, 2021

DOl: https://doi.org/10.21203/rs.3.rs-1046046/v1

License: (c) (i) This work is licensed under a Creative Commons Attribution 4.0 International License. Read Full License 


\section{Abstract}

Background: Bruton 's tyrosine kinase inhibitor ibrutinib has revolutionized the treatment of chronic lymphocytic leukemia (CLL). Although ibrutinib is a highly effective drug, continuous treatment is required to maintain remission, which may lead to acquired ibrutinib resistance. Early detection of acquired resistance preceding clinical disease progression is an important issue. This is why our aim was to investigate several phenotypic markers on CLL cells to reveal changes in their expression during ibrutinib treatment in sensitive and clinically resistant patients.

Materials and methods: In our study 28 (treatment naive, ibrutinib sensitive, clinically ibrutinib resistant) peripheral blood (PB), and 6 paired PB and bone marrow (BM) samples from CLL patients were examined. The expression of several surface markers (CD69, CD184, CD86, CD185, CD27) was assessed by flow cytometry in each sample. Furthermore, the presence of the BTK C481S resistance mutation was tested using digital droplet PCR (ddPCR) in samples from ibrutinib sensitive and resistant cases. In addition, we investigated the changes of CLL cells ' phenotype during ibrutinib treatment in one patient with acquired ibrutinib resistance.

Results: We found that the expression of CD27 decreased during ibrutinib therapy but increased again at the onset of clinical resistance. Expressions of CD69 and CD86 were also elevated at the onset of clinical ibrutinib resistance. Furthermore, the expression of CD86 showed correlation between PB and BM samples. Relapsed cases with high CD86 expression were positive for BTK C481S mutation. In addition, our prospective study showed that the increases in the expression of CD27, CD69 and CD86 were detectable up to several months before the onset of clinical resistance.

Conclusion: Our research suggests that the flow cytometric measurements of certain markers, especially CD86, may predict development of ibrutinib resistance, however, confirmatory experiments are still required. Monitoring CD86 expression on peripheral blood CLL cells during ibrutinib treatment may become a potential new method to detect acquired ibrutinib resistance in the near future.

\section{Background}

Chronic lymphocytic leukemia (CLL) is the most common type of leukemia in the western world [1, 2]. The irreversible Bruton's tyrosine kinase (BTK) inhibitor ibrutinib (IBR) shows remarkable efficiency in first-line as well in previously treated patients, therefore its role in CLL therapy is unquestionable $[3,4]$. Although ibrutinib is a highly effective drug, continuous treatment is required to maintain remission. In addition, appearance of drug resistance over time is a frequent and troublesome problem [5], making continuous treatment challenging. Point mutations of the BTK gene, such as the most frequent C481S missense mutation, prevent ibrutinib to bind to the kinase. Testing for the mutation is a well-known approach to predict resistance [6-8], however, the phenotypic changes following this process are poorly explored. Although the microenvironment may have a significant impact on ibrutinib resistance by providing survival factors [9-11], only a handful of researchers investigated it. Tissino et al. proved that CD49d 
could be a promising predictive factor of ibrutinib treatment. According to their data patients had shorter progression free survival if the CLL cells in peripheral blood were CD49d positive [12]. Since it is difficult to examine CLL cells in their microenvironment (e.g. in bone marrow (BM) or in the lymph nodes (LN)) in clinical setting, it would be useful to find markers on which expression on CLL cells in peripheral blood could predict the progression of the disease in their microenvironment as well. Considering this lack of scientific experience, in this study we aimed to examine further markers on peripheral blood CLL cells that play important roles in the pathogenesis of CLL (CD69, CD184, CD27, CD185, CD86) by flow cytometry [13-18]. Moreover, we examined whether the expression of these markers correlates with ibrutinib treatment or resistance.

\section{Materials And Methods \\ Patients and samples}

The investigated groups consisted of peripheral blood samples of treatment naive (Co) $(n=10$, female/male ratio 2/8; median age 73 years (57-85)), ibrutinib sensitive (IS) ( $n=7$ female/male ratio 5/2; median age 71 years (62-85)) and clinically ibrutinib-resistant (IR) ( $n=11$ female/male ratio 2/9; median age 70 years (56-87)) CLL patients. The diagnosis of CLL was based on the current WHO guideline [19]. Diagnosis of the clinical resistance was based on the current iwCLL guideline [20]. Patients in the ibrutinib sensitive group (IS) were treated with ibrutinib monotherapy for 1 year, and their samples were negative for $B T K^{C 481 S}$ mutation as determined by digital droplet PCR. The IR group patients have received ibrutinib monotherapy for 4-57 (median 25) months. We compared 6 paired BM and peripheral blood (PB) non-ibrutinib treated samples to detect potential compartmental differences. In the prospective study we observed changes in the expression of surface markers in the samples of an ibrutinib resistant patient not from the IR group. In this case salvage therapy was started with a $420 \mathrm{mg}$ daily dose of ibrutinib due to venetoclax resistance (day 0). We determined the expression of CD27, CD69 and CD86 on days 0, 30, $90,120,240,330$ and on the onset of the clinical resistance (day 420). Clinical parameters of this patient were published previously[21]. Patients were informed in writing and written consent was obtained from all participants. The study was approved by the Hungarian Medical Research Council (ID:453712/2016/EKU) and it was conducted in accordance with the Declaration of Helsinki.

\section{Flow cytometry}

Samples were measured using an 8-color Navios flow cytometer (Beckman Coulter (BC) FL, USA). Instrument settings were regularly controlled by Flow-Set Pro and Flow-Check Pro QC beads (BC). To obtain a satisfactory number of CLL cells, at least 50000 total events were acquired in each sample. The antibodies used in our experiments are listed in the Suppl. Table 1. Flow data were analyzed using Kaluza 2.1.1 software (BC). Living cells, lymphocytes, monocytes and granulocytes were identified based on side scatter (SSC) and CD45 dot-plot. B-cell to lymphocytes and T-cell to lymphocytes ratios were calculated based on CD19 and CD3 expressions. Finally, the proportion of CLL cells among CD19 positive 
lymphocytes was assessed based on CD5 expression. The CLL cell ratio among B-cells was above $98 \%$ in each sample, therefore CLL cells were considered as B-cells (Suppl. Fig. 1).

The method used to measure median fluorescence intensity (MFI) is based on our previously published work [21]. According to our measurements the CD19 negative lymphocyte population was suitable as an internal negative control for CD69, CD184, CD185 and CD86. Concerning CD27, the granulocyte population was used as internal negative control. Relative MFI values were calculated as the difference between the MFI value of internal negative controls and B-cells (Suppl. Fig. 2). These differences between the MFI values are displayed on the graphs.

\section{Detection of the $\mathrm{BTK}^{\mathrm{C} 481 \mathrm{~S}}$ resistance mutation}

$B T K^{\text {C481S }}$ resistance mutation was previously determined from the samples by digital droplet PCR (ddPCR; Bio-Rad Laboratories, CA, USA)[7, 8, 22]. In each sample, 100ng input DNA was used and all reactions were carried out according to the manufacturer's instructions. Droplets were generated by the QX200 Automated Droplet Generator followed by fluorescent signal detection using the QX200 Droplet Reader system. Results were evaluated and quantified using the Bio-Rad QuantaSoft software. The cutoff variant allele frequency (VAF) level was $0 \%$. Samples were considered positive for $B T K^{C 481 S}$ if the mutation was detected with a VAF higher than the $0 \%$ cut-off (Suppl. Fig. 3). BTK mutation status of all patients from the IR group was published previously by our research group [22].

\section{Statistical analysis}

We used SigmaPlot 12.5 (Systat Software Inc. CA, USA) for graphing and statistical analyses. Normality (Shapiro-Wilk) and equal variance tests were performed in each case, followed by t-tests or MannWhitney-tests based on their results. We performed linear regression to investigate correlations. Differences were considered statistically significant at $p<0.05$.

\section{Results}

\section{The impact of ibrutinib on CLL immunophenotype}

In our study we compared the immunophenotypes of treatment naive (Co), ibrutinib sensitive (IS) and clinically ibrutinib-resistant (IR) groups of CLL patients. We found that only the expression of CD27, CD69 and CD86 showed significant changes among the tested markers (Fig. 1). The expression of CD27 was significantly lower in the IS group than in the Co group (IS vs. Co: 51.136 vs. 169.114 MFI values, $p=0.003$ ). Furthermore, the expression of CD27 of CLL cells increased in IR cases compared to that of in the IS group (IS vs. IR: 51.136 vs. $156.341 \mathrm{MFI}$ values, $p=0.016$ ). In case of CD69 expression, significant difference was found only between the IS and IR groups. We observed that the expression of CD69 significantly increased in the IR cases (IS vs. IR: 9.92 vs. $97.24 \mathrm{MFI}$ values, $p=0.015$ ). The level of CD86 expression did not differ significantly between the IS and Co groups (IS vs. Co 27.23 vs. -9.84 MFI values, 
$\mathrm{p}=0.278$ ). However, CD86 expression was significantly increased in IR samples compared to IS (IR vs. IS 97.78 vs. $27.23 \mathrm{MFI}$ values, $p=0.015)$.

Unfortunately, we had no opportunity to follow up on any of the patients from the IR group, we could, however, observe how the expression of CD27, CD69 and CD86 are changing in the case of another ibrutinib resistant patient. We measured a baseline expression of the CD27, CD69 and CD86 at the beginning of ibrutinib treatment. Ibrutinib monotherapy seemed to be effective and the expression of all the three markers reached the lowest point by day 120 of treatment. From this timepoint expression of these markers continuously increased and by day 330 of treatment exceeded their baseline level (MFI values on day 330 vs. day 0: CD27: 218.26 vs. 200.42, CD69: 53.65 vs. 38.19, CD86: 165.07 vs. 148.39). Signs of clinical resistance (elevated absolute lymphocyte counts, enlarged lymph nodes) appeared on day 420 of treatment (Fig. 2.).

To reveal which marker represents trustworthily the immunophenotype of bone marrow CLL cells we compared 6 paired BM and PB samples. Only the expression of CD86 showed correlation in PB and BM, $(p=0.041, R=0.83$ ) (Suppl. Fig. 4.)

\section{Correlation between the $\mathrm{BTK}^{\mathrm{C} 481 S}$ resistance mutation and the immunophenotype of CLL cells}

To reveal a correlation between the $B T K^{C 481 S}$ mutation status and the immunophenotype of CLL cells during ibrutinib resistance, we assessed the mutation status of $B T K^{C 481 S}$ of ibrutinib resistant patients and the expression of the three selected markers (CD27, CD69, CD86). Among ibrutinib resistant samples 7 cases were positive for $B T K^{\mathrm{C} 481 \mathrm{~S}}$ mutation. Examining the expression of $\mathrm{CD} 27$ we found significant difference between $B T K^{C 481 S}$ positive and negative cases $\left(B T K^{\mathrm{C} 481 S}\right.$ positive vs. $B T K^{\mathrm{C} 481 \mathrm{~S}}$ negative 205.283 vs. $70.692 \mathrm{MFI}$ values, $p=0.011)$. In case of $C D 69$ we did not find any correlation $(p=0.176)$ between the expression of the marker and the $B T K^{\mathrm{C} 481 \mathrm{~S}}$ mutation status. Interestingly, the CD86 expression was significantly higher in samples with $B T K^{\mathrm{C} 481 \mathrm{~S}}$ mutation than in $B T K^{\mathrm{C} 481 \mathrm{~S}}$ negative samples ( $B T K^{\mathrm{C} 481 \mathrm{~S}}$ positive vs. $B T K^{\mathrm{C} 481 \mathrm{~S}}$ negative 134.28 vs. $33.92 \mathrm{MFI}$ values, $\left.\mathrm{p}<0.001\right)$ (Fig. 3.).

\section{Discussion}

Microenvironment is believed not only to play an important role in the pathogenesis of CLL but also to have an impact on drug resistance $[9,10,23]$. One of the most important off-target effects of ibrutinib is disrupting the tumor-supporting microenvironment, for example, by influencing the PD-1/PD-L1 pathway or restoring the impaired function of T-cells [24-26]. Although several microenvironmental immune functions related surface markers' expression have changed on CLL cells during ibrutinib therapy [11, 27], their expression are poorly investigated in the case of ibrutinib resistance. The change of expression of these markers may have an impact on the tumor immunity, leading to disease progression and drug resistance. Therefore, the research of the immune function related surface markers may help to reveal novel predictive markers in CLL. In our study, we compared the immunophenotypes of various groups of 
CLL patients (Co $(n=10)$, IS $(n=7)$ and IR $(n=11))$ by using 5 surface markers (CD69, CD184, CD86, CD185, $\mathrm{CD} 27)$ that are thought to be of great importance in the pathogenesis of CLL.

With respect to CD27 several studies have been carried out but their results seem to be controversial. While Shen et al. showed that the expression of CD27 increased on CLL cells during ibrutinib treatment [11], Rendeiro et al. detected the decrease of its expression upon the same treatment [27]. Our results confirmed Rendeiro's results. Namely, we found that the expression of CD27 on CLL cells significantly decreased during ibrutinib treatment compared to untreated samples. According to Riether et al. increased expression of CD27 on tumor cells may have an impact on the tumor supporting microenvironment. Therefore blocking CD27 may be a promising therapeutical target [28]. Based on literature there is a connection between the expression level of CD27 and the activation level of B-cell receptor signaling (BCR) pathway in CLL. Gobessi et al. proved that the BCR signaling pathway shows increased activity in Zeta Chain of T Cell Receptor Associated Protein Kinase 70 (ZAP70) positive CLL cells [29]. In addition Lafage et al. observed that the CD27 expression increased in ZAP70 ${ }^{+}$CLL cells [16]. Based on these two observations, increased expression of CD27 in CLL cells was accompanied by increased BCR activity hence it may be possible that the elevated CD27 expression is a sign of the increased activity of the BTK signaling pathway. In that case CD27 could be a novel biomarker for ibrutinib resistance. Therefore, we also compared the expression of CD27 on CLL cells between ibrutinib resistant and ibrutinib sensitive patient samples, and we found that its expression increased significantly in the case of ibrutinib resistance. It might look promising, but we do not have sufficient results to support this hypothesis, therefore further research is required.

CD69 may become a prognostic factor in CLL according to Del Poeta et al., who showed that the increased expression of CD69 could be a marker of worse prognosis in CLL [30]. It may explain the fact that CD69 is expressed on CLL cells as an activation marker and it plays a role in cell-cell interaction [13, 14]. Herman, et al. showed that the expression of CD69 decreases during ibrutinib treatment [31]. We found that the expression of CD69 was increased in ibrutinib resistance, therefore the expression of CD69 may be a useful resistance marker. Furthermore, we found that the expression of CD69 differs between BM and PB. Palma et al. observed a similar feature in correlation with PB and lymph node (LN) samples [32].

According to our results, CD86 seems to be the most promising candidate for a flow cytometric ibrutinib resistance marker among the tested molecules. Expression of CD86 on CLL cells is significantly lower than on non-tumor B-cells [33], and its expression increased on activated B-cells [34] suggesting that CD86 could be a marker for the activation of CLL cells $[35,36]$. The expression of CD86 may have a prognostic impact on CLL, because the increased expression of CD86 is associated with worse prognosis $[18,37]$. According to Herman et al. the expression of CD86 is decreased during ibrutinib treatment [31]. This result suggests that ibrutinib "repairs" the impaired immune functions in CLL [24], and this could be detected as a decrease in the expression level of activation markers. We explored the increased CD86 expression on CLL cells in ibrutinib resistant samples, suggesting that changes in the expression of CD86 are potential biomarkers for the monitoring the effectiveness of ibrutinib therapy and ibrutinib resistance. 
We also examined whether BTK mutation could be detected from ibrutinib resistant samples, and we found the expression of CD86 was significantly higher in $B T K^{C 481 S}$ mutant samples than in negative cases. Huemer et al. showed that the CD86 positive CLL cells have higher incidence of DNA damage [37]. This could lead to increased mutational burden, which may explain why $B T K^{\mathrm{C} 481 \mathrm{~S}}$ mutation is more frequent among CD86 positive CLL cells. According to literature, ibrutinib decreased the activation of CLL cells, which could be seen as a decrease in the expression of CD86 [31]. We showed that the expression of CD86 increased in ibrutinib resistant samples, suggesting that these CLL cells are re-activated by escaping the inhibitory effect of ibrutinib. Our prospective study revealed that the expression of CD27, CD69 and CD86 showed similar kinetics during ibrutinib treatment. Furthermore, these markers' expression level exceeded their baseline three months prior to the onset of clinical resistance. Based on our results, sensitive detection of $B T K^{C 481 S}$ may be a useful addition in case of increased CD86 expression. The measurement of CD86 expression by flow cytometry is a fast and simple method to detect ibrutinib resistance. Finally, expression levels of CD86 correlated between BM and PB samples, which raises the possibility of the expression of CD86 being independent from the CLL cells' anatomical localisation, but the clinical use of this observation is currently unclear.

\section{Conclusion}

Our results suggest that flow cytometric measurements of CD27, CD69 and CD86 may predict the emergence of ibrutinib resistance. Out of these markers, CD86 seems to be the most promising molecule as its expression on CLL cells did not differ between peripheral blood and bone marrow samples and a strong correlation was observable between its expression and the $B T K^{C 481 S}$ resistance mutation. CD86 may become a potentially new predictive marker of acquired ibrutinib resistance in the near future, although confirmatory experiments are still required.

\section{Declarations}

\section{Ethics approval and consent to participate:}

Patients were informed in writing and written consent was obtained from all participants. The study was approved by the Hungarian Medical Research Council (ID:45371-2/2016/EKU) and it was conducted in accordance with the Declaration of Helsinki.

\section{Acknowledgement:}

We would like to thank Zsolt Trembecki for English language editing and proofreading.

\section{Fundings}

This work was supported by the [Semmelweis University Directorate of Innovation] under Grant [STIA KF17/24/2017]; and [Hungarian National Research, Development and Innovation Office (NFKIH)] under 
Grant [EFOP-3.6.3-VEKOP-16-2017-00009] and EU's Horizon 2020 research and innovation program under grant agreement No. 739593.

\section{Conflict of interest}

The author's declare no conflist of interest.

\section{Availability of data and materials}

The datasets used and/or analysed during the current study are available from the corresponding author on reasonable request.

\section{Author's contributions:}

GB designed the study; GM, AM, PF, MP, JW, JD, LSz, BMD, Esz, AS and ZB provided patient samples and annotations; FT, LK, ÁC, FT, TL and ÁM performed experiments; FT, LK, ÁC, FT, TL, ÁM and SzG performed data analysis; FT, SzG and BG wrote the paper. All authors have read and critically reviewed the final version of the manuscript.

\section{References}

1. Zent, C.S., et al., Chronic lymphocytic leukemia incidence is substantially higher than estimated from tumor registry data. Cancer, 2001. 92(5): p. 1325-30.

2. Baumann, T., et al., Chronic lymphocytic leukemia in the elderly: clinico-biological features, outcomes, and proposal of a prognostic model. Haematologica, 2014. 99(10): p. 1599-604.

3. Burger, J.A., et al., Ibrutinib as Initial Therapy for Patients with Chronic Lymphocytic Leukemia. N Engl J Med, 2015. 373(25): p. 2425-37.

4. Woyach, J.A., et al., Ibrutinib Regimens versus Chemoimmunotherapy in Older Patients with Untreated CLL. N Engl J Med, 2018. 379(26): p. 2517-2528.

5. Ahn, I.E., et al., Clonal evolution leading to ibrutinib resistance in chronic lymphocytic leukemia. Blood, 2017. 129(11): p. 1469-1479.

6. Burger, J.A., et al., Clonal evolution in patients with chronic lymphocytic leukaemia developing resistance to BTK inhibition. Nat Commun, 2016. 7: p. 11589.

7. Gángó, A., et al., Dissection of subclonal evolution by temporal mutation profiling in chronic lymphocytic leukemia patients treated with ibrutinib. Int J Cancer, 2020. 146(1): p. 85-93.

8. Kiss, R., et al., Spatial clonal evolution leading to ibrutinib resistance and disease progression in chronic lymphocytic leukemia. Haematologica, 2019. 104(1): p. e38-e41. 
9. Boissard, F., et al., Nurse-like cells mediate ibrutinib resistance in chronic lymphocytic leukemia patients. Blood Cancer J, 2015. 5: p. e355.

10. Guo, A., et al., Dual SYK/JAK inhibition overcomes ibrutinib resistance in chronic lymphocytic leukemia: Cerdulatinib, but not ibrutinib, induces apoptosis of tumor cells protected by the microenvironment. Oncotarget, 2017. 8(8): p. 12953-12967.

11. Shen, Y., et al., Ibrutinib and idelalisib block immunophenotypic changes associated with the adhesion and activation of CLL cells in the tumor microenvironment. Leuk Lymphoma, 2018. 59(8): $\mathrm{p}$. 1927-1937.

12. Tissino, E., et al., Functional and clinical relevance of VLA-4 (CD49d/CD29) in ibrutinib-treated chronic lymphocytic leukemia. J Exp Med, 2018. 215(2): p. 681-697.

13. Damle, R.N., et al., B-cell chronic lymphocytic leukemia cells express a surface membrane phenotype of activated, antigen-experienced B lymphocytes. Blood, 2002. 99(11): p. 4087-93.

14. Montraveta, A., et al., CD69 expression potentially predicts response to bendamustine and its modulation by ibrutinib or idelalisib enhances cytotoxic effect in chronic lymphocytic leukemia. Oncotarget, 2016. 7(5): p. 5507-20.

15. Ganghammer, S., et al., Combined CXCR3/CXCR4 measurements are of high prognostic value in chronic lymphocytic leukemia due to negative co-operativity of the receptors. Haematologica, 2016. 101(3): p. e99-102.

16. Lafarge, S.T., et al., Differential expression and function of CD27 in chronic lymphocytic leukemia cells expressing ZAP-70. Leuk Res, 2015. 39(7): p. 773-8.

17. Sáez de Guinoa, J., et al., CXCL 13/CXCR5 signaling enhances BCR-triggered B-cell activation by shaping cell dynamics. Blood, 2011. 118(6): p. 1560-9.

18. Takács, F., et al., The Effect of CD86 Expression on the Proliferation and the Survival of CLL Cells. Pathol Oncol Res, 2019. 25(2): p. 647-652.

19. WHO Classification of Tumours of Haematopoietic and Lymphoid Tissues. 4th ed. World Health Organization Classification of Tumours, ed. F.T. Bosman, et al. 2017, Lyon: International Agency for Research on Cancer.

20. Hallek, M., et al., iwCLL guidelines for diagnosis, indications for treatment, response assessment, and supportive management of CLL. Blood, 2018. 131(25): p. 2745-2760.

21. Takács, F., et al., Identification of a novel resistance mechanism in venetoclax treatment and its prediction in chronic lymphocytic leukemia. Acta Oncol, 2021: p. 1-3. 
22. Bodor, C., et al., Screening and monitoring of the BTK(C481S) mutation in a real-world cohort of patients with relapsed/refractory chronic lymphocytic leukaemia during ibrutinib therapy. $\mathrm{Br} \mathrm{J}$ Haematol, 2021. 194(2): p. 355-364.

23. Herishanu, Y., et al., The lymph node microenvironment promotes B-cell receptor signaling, NFkappaB activation, and tumor proliferation in chronic lymphocytic leukemia. Blood, 2011. 117(2): p. 56374.

24. Long, M., et al., Ibrutinib treatment improves $T$ cell number and function in CLL patients. J Clin Invest, 2017. 127(8): p. 3052-3064.

25. Kondo, K., et al., Ibrutinib modulates the immunosuppressive CLL microenvironment through STAT3-mediated suppression of regulatory B-cell function and inhibition of the PD-1/PD-L 1 pathway. Leukemia, 2018. 32(4): p. 960-970.

26. Niemann, C.U., et al., Disruption of in vivo Chronic Lymphocytic Leukemia TumorMicroenvironment Interactions by Ibrutinib--Findings from an Investigator-Initiated Phase II Study. Clin Cancer Res, 2016. 22(7): p. 1572-82.

27. Rendeiro, A.F., et al., Chromatin mapping and single-cell immune profiling define the temporal dynamics of ibrutinib response in CLL. Nat Commun, 2020. 11(1): p. 577.

28. Riether, C., C. Schürch, and A.F. Ochsenbein, Modulating CD27 signaling to treat cancer. Oncoimmunology, 2012. 1(9): p. 1604-1606.

29. Gobessi, S., et al., ZAP-70 enhances B-cell-receptor signaling despite absent or inefficient tyrosine kinase activation in chronic lymphocytic leukemia and lymphoma B cells. Blood, 2007. 109(5): p. 2032-9.

30. Del Poeta, G., et al., CD69 is independently prognostic in chronic lymphocytic leukemia: a comprehensive clinical and biological profiling study. Haematologica, 2012. 97(2): p. 279-87.

31. Herman, S.E., et al., Ibrutinib inhibits BCR and NF-KB signaling and reduces tumor proliferation in tissue-resident cells of patients with CLL. Blood, 2014. 123(21): p. 3286-95.

32. Palma, M., et al., Ibrutinib induces rapid down-regulation of inflammatory markers and altered transcription of chronic lymphocytic leukaemia-related genes in blood and lymph nodes. Br J Haematol, 2018. 183(2): p. 212-224.

33. Dai, Z.S., et al., Defective expression and modulation of B7-2/CD86 on B cells in B cell chronic lymphocytic leukemia. Int J Hematol, 2009. 89(5): p. 656-63.

34. Axelsson, S., et al., A combination of the activation marker CD86 and the immune checkpoint marker $B$ and $T$ lymphocyte attenuator (BTLA) indicates a putative permissive activation state of $B$ cell subtypes in healthy blood donors independent of age and sex. BMC Immunol, 2020. 21(1): p. 14. 
35. Burger, J.A., et al., The microenvironment in mature B-cell malignancies: a target for new treatment strategies. Blood, 2009. 114(16): p. 3367-75.

36. Lim, T.S., et al., CD80 and CD86 differentially regulate mechanical interactions of T-cells with antigen-presenting dendritic cells and B-cells. PLoS One, 2012. 7(9): p. e45185.

37. Huemer, M., et al., AID induces intraclonal diversity and genomic damage in CD86(+) chronic lymphocytic leukemia cells. Eur J Immunol, 2014. 44(12): p. 3747-57.

\section{Figures}

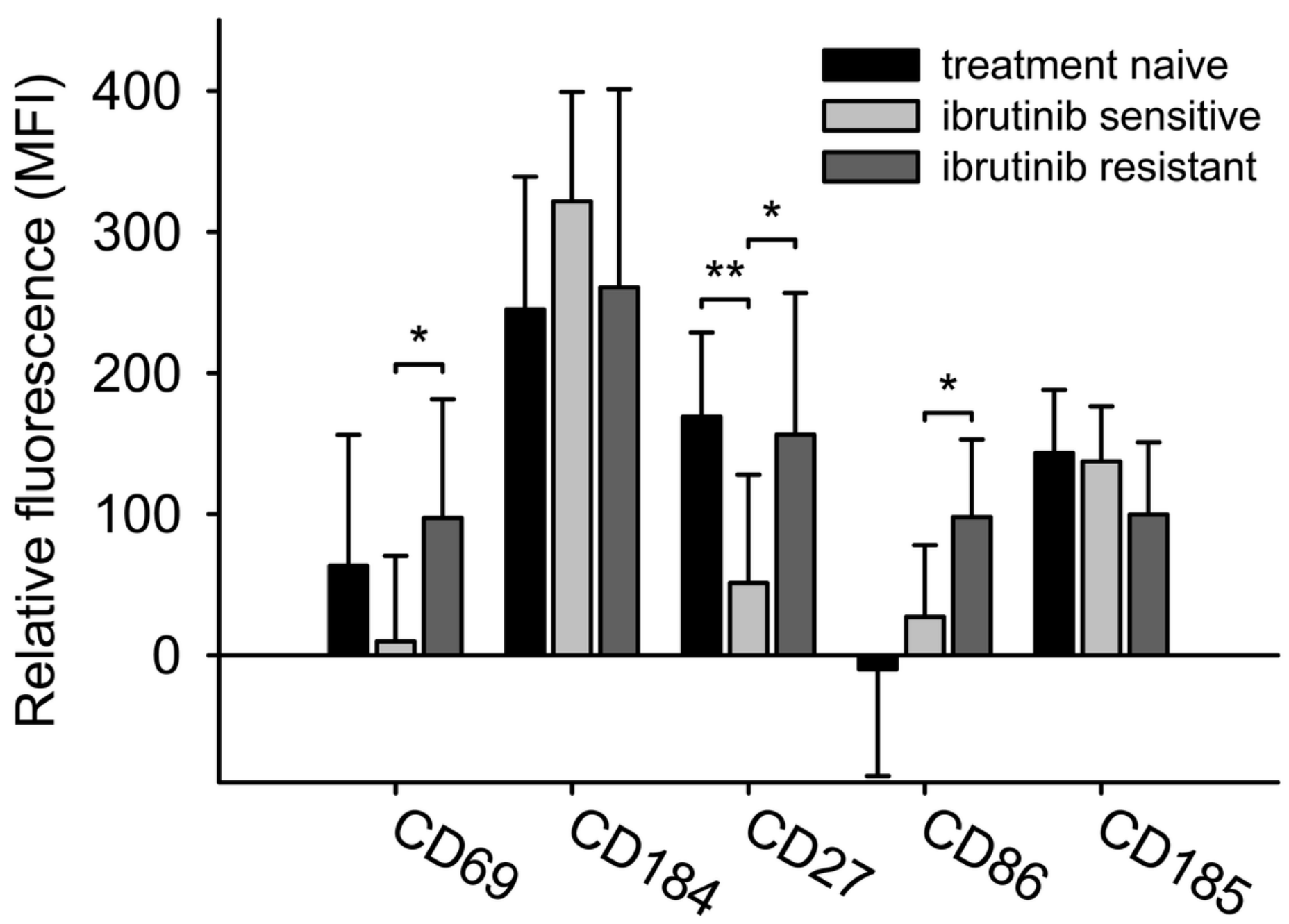

Figure 1

The impact of ibrutinib on CLL cell's immunophenotype We found that among the investigated markers only CD27, CD69, and CD86 expressions showed significant changes in the treatment naive (Co, $n=10)$, 
ibrutinib sensitive (IS, n=7) and ibrutnib resistant (IR, $n=11)$ groups. The expression of CD27 was significantly lower in the IS group than in the Co group. Furthermore, there was also a significant difference between the IS and IR groups. We observed that the expression of CD69 was significantly elevated in the IR group as compared to IS group. In the case of CD86 expression,, we observed no significant difference between the Co and IS groups, however its expression was significantly elevated in the IR group compared to the IS. ${ }^{*} \mathrm{P}<0.05 ; * * \mathrm{P}<0.01$

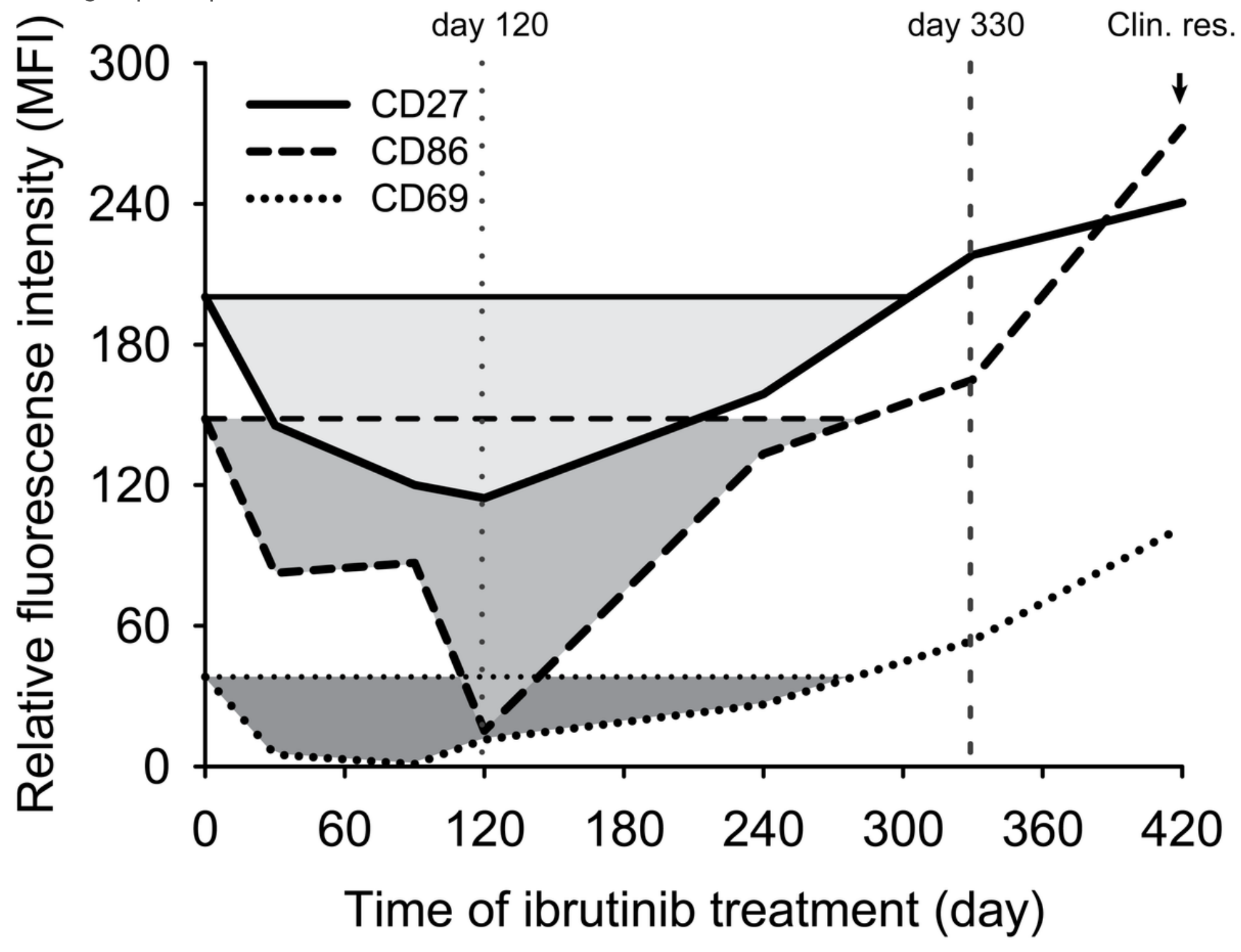

Figure 2

The kinetics of investigated markers during ibrutinib treatment The graph highlights the kinetics of investigated markers during ibrutinib treatment in a resistant case. Ibrutinib salvage therapy was started with a $420 \mathrm{mg}$ daily dose due to venetoclax resistance (day 0 ). We assessed the expression levels of CD27, CD69 and CD86 on day $0,30,90,120,240,330$ and on the onset of the clinical resistance on day 420. We concluded that all markers showed similar kinetics during ibrutinib treatment. Furthermore, the increased expression of CD27, CD69, and CD86 were detectable months prior to the the onset of clinical resistance (Clin. res.). 


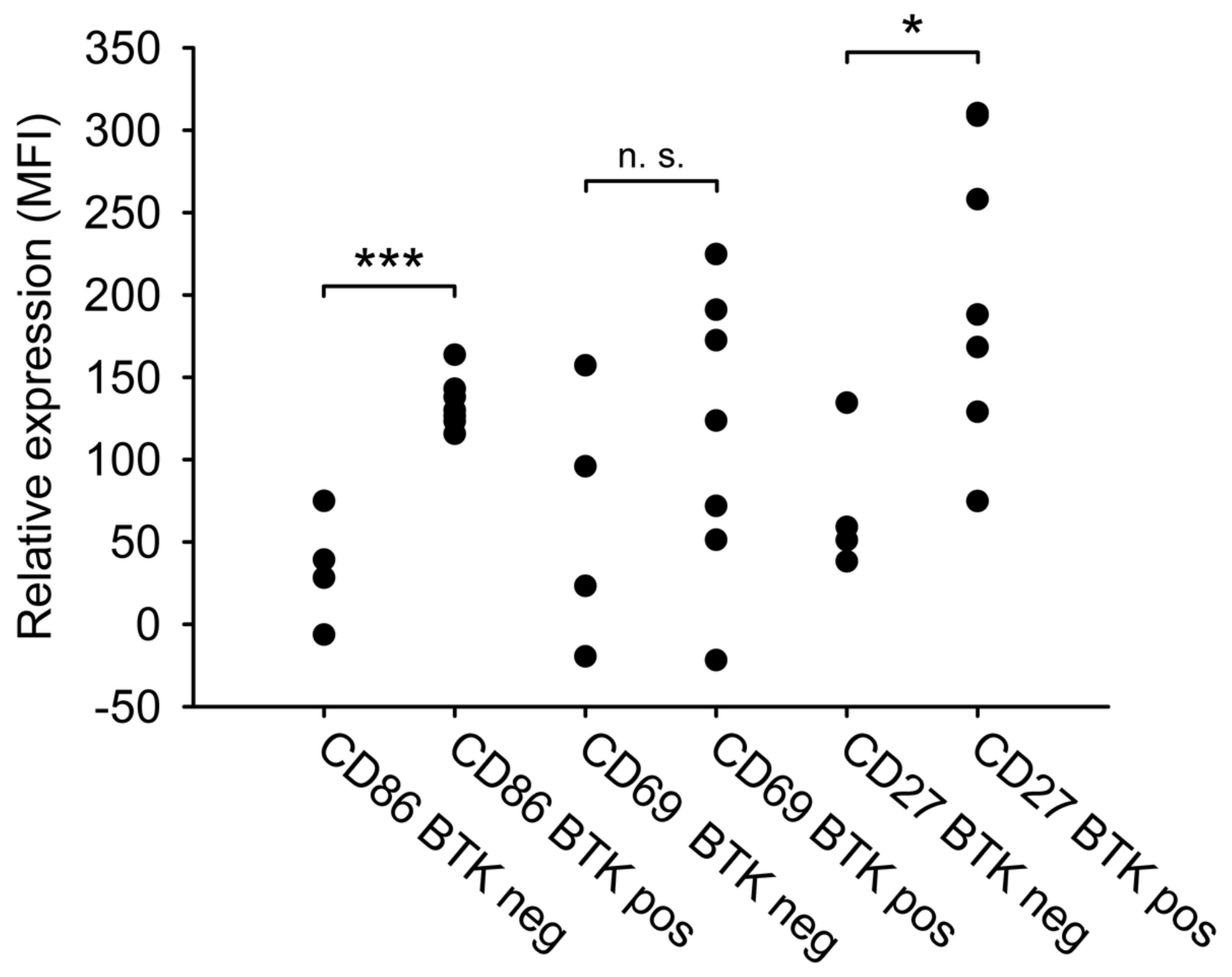

Figure 3

Correlation between BTKC481S resistance mutation and the immunophenotype of CLL cells in clinically ibrutinib resistant cases The graph shows the connection between the BTKC481S mutation status and phenotypic markers in case of clinically resistant patients. Patients were divided into two groups based on CD86 expression. In the case of BTKC481S mutation CD86 expression was significantly higher than in BTKC481S negative samples. In case of CD69, we did not find any correlation. In terms of CD27 we found a significant difference between BTKC481S positive and negative groups, although our data showed big dispersion. ${ }^{*} \mathrm{P}<0.05 ; * \star * \mathrm{P}<0.001$

\section{Supplementary Files}

This is a list of supplementary files associated with this preprint. Click to download.

- supplementarydata.docx 